\title{
Modeling skin sensitization potential of mechanistically hard-to-be-classified aniline and phenol compounds with quantum mechanistic properties
}

Qin Ouyang ${ }^{1,4+}$, Lirong Wang ${ }^{1 \dagger}$, Ying $\mathrm{Mu}^{3}$ and Xiang-Qun Xie ${ }^{1,2^{*}}$

\begin{abstract}
Background: Advanced structure-activity relationship (SAR) modeling can be used as an alternative tool for identification of skin sensitizers and in improvement of the medical diagnosis and more effective practical measures to reduce the causative chemical exposures. It can also circumvent ethical concern of using animals in toxicological tests, and reduce time and cost. Compounds with aniline or phenol moieties represent two large classes of frequently skin sensitizing chemicals but exhibiting very variable, and difficult to predict, potency. The mechanisms of action are not well-understood.
\end{abstract}

Methods: A group of mechanistically hard-to-be-classified aniline and phenol chemicals were collected. An in silico model was established by statistical analysis of quantum descriptors for the determination of the relationship between their chemical structures and skin sensitization potential. The sensitization mechanisms were investigated based on the features of the established model. Then the model was utilized to analyze a subset of FDA approved drugs containing aniline and/or phenol groups for prediction of their skin sensitization potential.

Results and discussion: A linear discriminant model using the energy of the highest occupied molecular orbital $\left(\epsilon_{\text {HOMO }}\right)$ as the descriptor yielded high prediction accuracy. The contribution of $\epsilon_{\text {HOMO }}$ as a major determinant may suggest that autoxidation or free radical binding could be involved. The model was further applied to predict allergic potential of a subset of FDA approved drugs containing aniline and/or phenol moiety. The predictions imply that similar mechanisms (autoxidation or free radical binding) may also play a role in the skin sensitization caused by these drugs.

Conclusions: An accurate and simple quantum mechanistic model has been developed to predict the skin sensitization potential of mechanistically hard-to-be-classified aniline and phenol chemicals. The model could be useful for the skin sensitization potential predictions of a subset of FDA approved drugs.

Keywords: Chemical mechanisms, Structure-activity relationship, Skin sensitizer, Anilines, Phenols, Quantum mechanism

\footnotetext{
* Correspondence: xix15@pitt.edu

${ }^{\dagger}$ Equal contributors

'Department of Pharmaceutical Sciences, Computational Chemical Genomics Screening Center, School of Pharmacy, NIH National Center, of Excellence for Computational Drug Abuse Research, Drug Discovery Institute, Pittsburgh, PA 15261, USA

${ }^{2}$ Department of Computational and Systems Biology, University of Pittsburgh, Pittsburgh, PA 15261, USA

Full list of author information is available at the end of the article
}

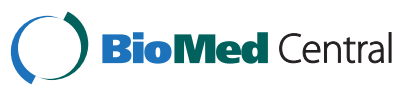

(c) 2014 Ouyang et al.; licensee BioMed Central. This is an Open Access article distributed under the terms of the Creative Commons Attribution License (http://creativecommons.org/licenses/by/4.0), which permits unrestricted use, distribution, and reproduction in any medium, provided the original work is properly credited. The Creative Commons Public Domain Dedication waiver (http://creativecommons.org/publicdomain/zero/1.0/) applies to the data made available in this article, unless otherwise stated. 


\section{Background}

Skin sensitization related dermatitis and rash represents the most common manifestation of chemical immunotoxicity in humans, which results in a cost estimated $\$ 1$ billion annually due to lost work, reduced productivity, medical care, and disability payments in USA [1,2]. In addition, as part of the regulatory review process, an increase in the incidence of skin allergies and hypersensitivity-related adverse events associated with the use of FDA regulated products or approved drugs has been observed, suggesting a safety gap between premarket review and the post market surveillance [2].

Common testing methods to assess skin sensitization potential of materials include: (1) guinea pig maximization test (GPMT); (2) murine-based local lymph node assay (LLNA). In GPMT tests, hazard identification is done by visual observations of erythema and edema reactions, which are subjective, are difficult to differentiate between contact allergens and strong irritants, and is time consuming [3]. The LLNA is recommended by international regulatory agencies; however, inconsistencies between LLNA and clinical observations have been documented [4]. Considering the existence of vast compounds around today, developing rapid and effective methods for chemical sensitizer identification/risk assessment is still a challenge [2].

In silico approaches are an attractive alternative to animal testing through analyzing the structural features of sensitizers/non-sensitizers to derive predictive rules or models [5]. The risks of thousands of commercially available chemicals could be assessed in a cost effective manner. Among these approaches, mechanism based rules, which investigate the structural characteristics of sensitizers, are promising [6].

Historically, the first study of chemical reactivity and skin sensitization was reported in 1936 [7]. A mechanism of small organic molecules to form an immunogenic complex by reacting with macromolecules (proteins or others) in the skin to cause sensitization was postulated. Currently, a more plausible mechanism reported involves a formation of covalent bonding between electrophilic allergens and nucleophilic moieties of amino acids from skin proteins (usually side chains) [8]. Such amino acids include cysteine thiol (mainly) and lysine (amino), and to a lesser extent arginine, histidine, methionine and tyrosine [9]. Based on the well-established principles of mechanistic organic chemistry, the skin sensitization potential of a chemical in many cases was predicted by its reactivity with these residues $[9,10]$. However, some compounds need to be activated via either autoxidation outside the skin (prehaptens) or bioactivation inside the skin (prohaptens) to be able to form immunogenic complexes with skin proteins [11].

Structure-activity relationship (SAR) studies of skin sensitization potential have been successfully carried out for epoxyaldehydes [12], enone [13], halogenated aromatics [14], benzaldehydes [15], dienes [16], oximes [17], aldehydes [18] and epoxides [19]. Aniline/aromatic amine and/or phenol derivatives are two large classes of frequently sensitizing chemicals. Quite a few pilot studies have been conducted [20-23]. Roberts et al. specifically investigated the sensitization mechanisms of diaminobenzenes or dihydroxylbenzenes [24]. However, the predictability of the skin sensitization potential for these two classes of chemicals is unsatisfactory $[6,25]$. Further exploration of novel sensitization mechanisms will be informative for constructing better SAR models/rules. In addition, aniline and phenol moieties that are often present in approved drugs can also cause skin sensitization. For example, contact dermatitis occurs in one individual following prolonged subcutaneous infusion of hydromorphone [26], a cancer pain treatment agent which contains one phenol moiety.

Drug-induced skin reactions may be associated with several biological mechanisms, but in many cases the precise mechanism is unclear [27]. It is well-known that Type IV allergic reaction induced by many chemicals and drugs is a T-cell mediated delay type hypersensitivity which can cause skin sensitization/dermatitis [27].

In this study, we intended to establish an in silico model for a class of mechanistically hard-to-classify anilines and phenols to study the relationship between their chemical reactivity and biological allergic response. We then investigated sensitization mechanisms of action associated with these compounds based on the features of this model. The model was further utilized to analyze a subset of FDA approved drugs containing aniline and/or phenol groups in skin sensitization potential. The predicted skin sensitization potential for these drugs was validated according to relevant literatures and adverse event reports.

\section{Methods}

\section{Data sets}

A data set of 63 chemicals, including 30 anilines and 33 phenols, was collected from published literature [11,23,28-36]. Chemicals with well-known allergic mechanisms, i.e. Michael acceptors (MA), $\mathrm{SN}_{2}$ electrophiles, $\mathrm{S}_{\mathrm{N}} \mathrm{Ar}$ electrophiles, Schiff base formers, and acylation agents, were excluded from the data set. For example, pentachlorophenol (CAS: 87-86-5) belongs to $\mathrm{S}_{\mathrm{N}} \mathrm{Ar}$ electrophiles; benzyl salicylate (CAS: 118-58-1) and 3,3',4',5tetrachlorosalicylanilide (CAS: 1154-59-2) are acylation agents. In addition, chemicals having two $\mathrm{OH}$ and $\mathrm{NH}_{2}$ substituents at aromatic rings were also excluded from the data set because these compounds are known to easily form a benzoquinone (a Michael acceptor) or a nitrogen analogue of benzoquinone (also a Michael acceptor) [24]. Finally, a list of 30 chemicals was obtained (Table 1). They 
Table 1 Summary of the $\epsilon_{\text {Hомо }}$ predicted values of the $\mathbf{3 0}$ chemicals and their experimentally determined data $[11,23,28-36]$

\begin{tabular}{|c|c|c|c|c|c|c|c|}
\hline ID & Cas \# & Name & Sensitizer & EC3 & Ref. & $\epsilon_{\text {номо }}$ (hartree) & $P$ \\
\hline 1 & $101-80-4$ & 4,4-diaminodiphenylether & $\mathrm{Y}$ & & 30 & -0.262 & 1.0694 \\
\hline 2 & $106-47-8$ & 4-Chloroaniline & Y & 6.5 & 33,35 & -0.289 & 0.6563 \\
\hline 3 & $150-13-0$ & 4-Aminobenzoic acid & N & & 31 & -0.306 & 0.3962 \\
\hline 4 & $369-36-8$ & 2-Fluoro-5-nitroaniline & N & & 34 & -0.325 & 0.1055 \\
\hline 5 & $538-41-0$ & 4,4-diaminoazobenzene & Y & & 30 & -0.258 & 1.1306 \\
\hline 6 & $62-53-3$ & Aniline & Y & 89 & 31,36 & -0.286 & 0.7022 \\
\hline 7 & $79456-26-1$ & 3-Chloro-5-(trifluoromethyl)-2-pyridinamine & N & & 23 & -0.326 & 0.0902 \\
\hline 8 & $2050-14-8$ & 2,2'-Azodiphenol & Y & 27.9 & 34 & -0.294 & 0.5798 \\
\hline 9 & $55845-90-4$ & (N-Benzyl-N-ethylamino)-3'-hydroxyacetophenone hydrochloride & N & & 23 & -0.316 & 0.2432 \\
\hline 10 & $69-72-7$ & Salicylic acid & N & & 31 & -0.343 & -0.1699 \\
\hline 11 & $90-15-3$ & 1-Naphthol & Y & 1.3 & $11,29.33$ & -0.274 & 0.8858 \\
\hline 12 & $94-13-3$ & Propylparaben & N & & 11 & -0.329 & 0.0443 \\
\hline 13 & $97-54-1$ & Isoeugenol & Y & 3.5 & $31,33,32$ & -0.277 & 0.8399 \\
\hline 14 & $99-96-7$ & 4-Hydrobenzoic acid & N & & 11 & -0.341 & -0.1393 \\
\hline 15 & $186743-26-0$ & 3-Methyleugenol & Y & 32 & 33,36 & -0.294 & 0.5798 \\
\hline 16 & 101-77-9* & 4,4-diaminodiphenylmethane & Y & & 30 & -0.266 & 1.0082 \\
\hline 17 & $121-57-3^{*}$ & Sulphanilic acid & N & & 11 & -0.322 & 0.1514 \\
\hline 18 & $537-65-5^{*}$ & 4,4-diaminodiphenylamine & Y & & 30 & -0.243 & 1.3601 \\
\hline 19 & $60-09-3^{*}$ & 4-aminophenylazobenzene & Y & & 30 & -0.276 & 0.8552 \\
\hline 20 & $63-74-1^{*}$ & Sulfanilamide & N & & 11 & -0.307 & 0.3809 \\
\hline 21 & $94-09-7^{*}$ & Benzocaine & N & & 33 & -0.303 & 0.4421 \\
\hline 22 & $15128-82-2^{*}$ & 3-Hydroxy-2-nitropyridine & N & & 31 & -0.362 & -0.4606 \\
\hline 23 & $2785-87-7^{*}$ & Dihydroeugenol & Y & 12.45 & $11,29,32$ & -0.288 & 0.6716 \\
\hline 24 & $619-14-7^{*}$ & 3-Hydroxy-4-nitrobenzoic acid & N & & 31 & -0.371 & -0.5983 \\
\hline 25 & $80-05-7^{*}$ & bisphenol A & Y & & 30 & -0.287 & 0.6869 \\
\hline 26 & $93-51-6^{*}$ & 2-Methoxy-4-methyl-phenol & Y & 5.8 & $11,29,32$ & -0.288 & 0.6716 \\
\hline 27 & $97-53-0^{*}$ & Eugenol & Y & 13.95 & $31,32,33$ & -0.296 & 0.5492 \\
\hline 28 & $99-76-3^{*}$ & Methyl 4-hydroxybenzoate & $N$ & & 11 & -0.329 & 0.0443 \\
\hline 29 & $119-36-8^{*}$ & Methyl salicylate & $\mathrm{N}$ & & 28 & -0.326 & 0.0902 \\
\hline 30 & $831-82-3^{*}$ & 4-Phenoxyphenol & Y & & 33 & -0.293 & 0.5951 \\
\hline
\end{tabular}

*Test set.

represent a class of mechanistically hard-to-be-classified compounds because they can't be classified into any of the abovementioned five categories. These compounds were then randomly split into a training set of 15 compounds and a test set of 15 compounds. As shown in Table 1, the training set includes 7 anilines and 8 phenols, while the test set includes 6 anilines and 9 phenols. The detailed information including initial screening of the 63 selected chemicals is available as Additional file 1.

\section{Quantum mechanics calculations}

All chemical optimization and subsequent orbital analysis were performed by using the Gaussian 03 suite of programs [37]. Chemicals were optimized using the
AM1 Hamiltonian with the default optimization criteria [38,39]. Calculations of the frontier molecular orbital, charge distribution and other quantum properties were carried out by using the 6-31Gd basis set. The quantum descriptors used in this study include the energies of the highest occupied molecular orbital $\left(\epsilon_{\mathrm{HOMO}}\right)$, the lowest unoccupied molecular orbital $\left(\epsilon_{\mathrm{LUMO}}\right)$, the second lowest unoccupied molecular orbital $\left(\epsilon_{\mathrm{LUMO}+1}\right)$, the second highest occupied molecular orbital $\left(\epsilon_{\mathrm{HOMO}-1}\right)$, the Mulliken atomic charges of the most negative $\left(Q_{\min }\right)$ and most positive atoms $\left(Q_{\max }\right)$, the Mulliken atomic charges of the $\mathrm{N}$ atom $\left(Q_{\mathrm{N}}\right)$ in anilines or $\mathrm{O}$ atom $\left(Q_{\mathrm{O}}\right)$ in phenols, the average of the absolute values of the charges on all atoms $\left(Q_{\mathrm{m}}\right)$, and molecular dipole moment $(\mu)$. The 
shapes of the resulting orbitals were visualized using the GaussView application within Gaussian 03. All structures were either drawn or converted from SMILES (Simplified molecular-input line-entry system) strings, using Chembiodraw Ultra V12.0 (PerkinElmer Informatics Desktop Software).

\section{Statistical analysis}

The skin sensitization potency of a compound was symbolized by 1 (Yes) and 0 (No). The values of each quantum descriptor were linearly normalized to the same range $(0$ to 1), stepwise linear regressions between the quantum properties and experimental outputs of the training set were performed by the statistical package of $\mathrm{R}$ program version 3.0.0 [40]. The properties with lower weighting factors were abandoned in the second step of linear regression.

\section{Results and discussion}

The compounds with aniline and/or phenol moieties can be classified into a single subclass for consideration of skin sensitizers. However, not all of the compounds possessing aniline or phenol groups are sensitizers, suggesting some compounds can form covalent bonds with skin proteins whereas others cannot. In this study, the sensitization potential of anilines and phenols were modeled using quantum mechanical descriptors.

\section{Modeling the skin sensitization potential by quantum properties of anilines and phenols}

The coefficient constant of $\epsilon_{\text {HOMO }}$ was determined as the highest weighting factor based on the results of linear regression analysis. The skin sensitization potential of anilines and phenols can be formulated as:

$$
\begin{aligned}
\text { Predicted Value }(P)= & 15.30 * \epsilon_{\text {HOMO }}(\text { Hartree }) \\
& +5.08(1)
\end{aligned}
$$

The median of the symbolized skin sensitization potency, 0.50, was considered as the threshold for prediction of sensitizers and non-sensitizers. An aniline or phenol is predicted as a sensitizer if $P$ is greater than 0.50 , and as a non-sensitizer if $P$ is below 0.5 . With a threshold of $\mathrm{P}=0.50$, Equation 1 implies that a chemical within the applicability domain is predicted to be a skin sensitizer if the HOMO energy is greater than -0.30 Hartree $((0.5-5.08) / 15.30=-0.299 \approx-0.30)$. The experimental allergenicity categories (sensitizer or non-sensitizer) and predicted results of the training set are shown in Figure 1, where red-open circle dots indicate wellknown sensitizers at 1 and non-sensitizers at 0 , respectively. The blue-solid-diamond dots indicate the predicted values. All of the training compounds were correctly predicted by Formula 1. The same model was applied to the

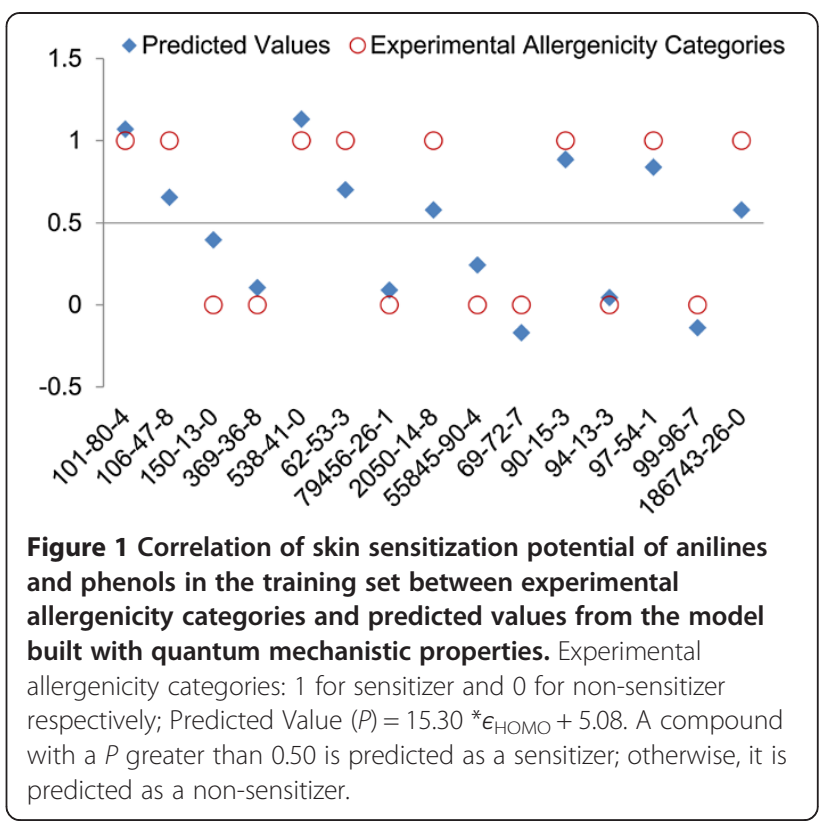

test set. Interestingly, all test compounds were correctly predicted (Figure 2). The total prediction accuracy of chemicals in training and test sets was 100\% (30/30). The model shows very high accuracy and only depends on the value of $\epsilon_{\mathrm{HOMO}}$, suggesting that $\epsilon_{\mathrm{HOMO}}$ is a key factor for the assessment of skin sensitization potential of those aniline and phenol containing compounds.

The linear relationship between the predicted values and $\epsilon_{\mathrm{HOMO}}$ also suggests that a chemical with higher predicted value implies a higher reactivity for oxidation consequently resulting in higher skin sensitization potential. The LLNA data as a quantitative endpoint, posed

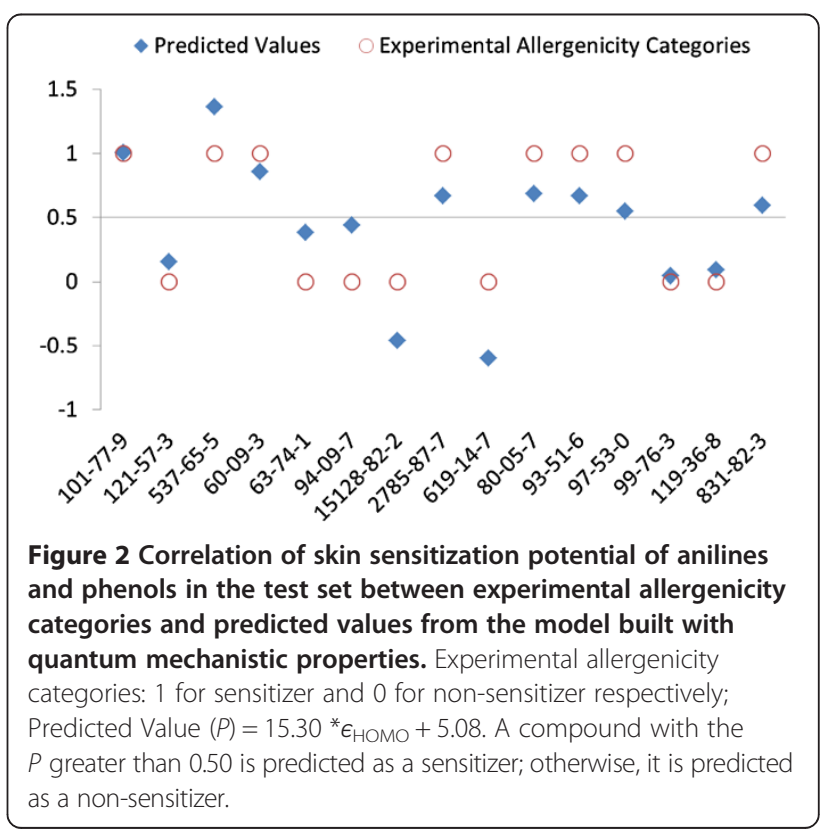


a semi-dose-dependent manner, allows for prediction of potency. The EC3 values (effective concentration for a three-fold proliferation of lymph node cells) from the reported LLNA experiments of most allergen phenols were also collected as shown in Table 1 . Weak sensitizers with higher EC3 values (meaning lower sensitization potential) have smaller $P$ values. For example, $P$ values of five weak sensitizers with $100>\mathrm{EC} 3>10$ i.e., eugenol (CAS: 97-53-0, EC3 = 13.95), Dihydroeugenol (CAS: 2785-87-7, EC3 $=12.45$ ), 2,2'-azodiphenol (CAS: 2050-14-8, EC3 = 27.90), 3-methyleugenol (CAS: 186743-26-0,EC3 = 32), and aniline (CAS: $62-53-3, \mathrm{EC} 3=89$ ) were $0.549,0.672$, $0.580,0.580$, and 0.702 , respectively. On the other hand, two moderate sensitizers, 2-Methoxy-4-methyl-phenol (CAS: 93-51-6, EC3 = 5.8), 4-Chloroaniline (CAS: 106$47-8$, EC3 $=6.5$ ), have a slightly higher $P$ value 0.672 , 0.656 , respectively. Another two moderate sensitizers with smaller EC3 value, isoeugenol (CAS: 97-54-1, EC3 = 3.5) and 1-naphthol (CAS: 90-15-3, EC3 =1.3) have greater $P$ values, 0.840 and 0.886 , respectively. For most chemicals, their - $\operatorname{logEC} 3$ values correlate with $P$ values quite well, but for aniline, its $-\operatorname{logEC} 3$ value is much less potent than its $P$ value predicted. This may indicate that the initial oxidation of aniline, which is quite fast, is not in this case the rate-determining step for protein haptenation. The analysis of the relationship between EC3 and

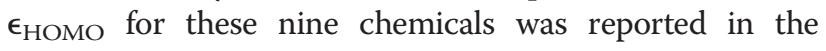
Additional file 1.

\section{Possible reaction mechanisms of aromatic anilines and phenols}

Occurrence of electrophilic-nucleophilic reactions between chemical and skin proteins is a primary reason of chemical induced skin sensitization [8]. Most chemicals with high skin sensitization potential can be classified as Michael acceptors (MA), $\mathrm{SN}_{2}$ electrophiles, $\mathrm{S}_{\mathrm{N}}$ Ar electrophiles, Schiff base formers, or acylation agents. The reaction mechanisms of anilines and phenols, however, are poorly understood and very few of them can be classified into the aforementioned five categories. One proposed mechanism is that sensitization occurs via oxygen attack ortho to an amino group or via oxidative quinonemethide formation [25,41]. For example, Roberts et al. reported the mechanistic chemistry of aromatic diamino-, dihydroxy-, and amino-hydroxy compounds [24] where two parallel chemical mechanisms were described as the most possible processes: oxidation to electrophilic (protein reactive) quinones, quinone imines, or quinone di-imines or formation of protein reactive free radicals. These mechanisms, unfortunately, are not applicable to the all single $\mathrm{NH} / \mathrm{OH}$ substituted anilines and phenols. For instance, aniline and 4-butylaniline are sensitizers whereas 4aminobenzoic acid, 4-aminobenzenesulfonamide, and 4aminobenzenesulfonic acid are non-sensitizers. Beside the solubility effects and the formation of ions/zwitterions, the reactivity variety of chemical entities by substituent effects play an important role in reducing dermal penetration and immunogenicity of protein conjugates.

By analyzing the relationship between quantum properties and chemical reactivity, we successfully modeled the skin sensitization potential of two groups of chemicals (aromatic anilines and phenols) with a single coefficient of $\epsilon_{\mathrm{HOMO}}$, while the energy of the lowest unoccupied molecular orbital $\left(\epsilon_{\text {LUMO }}\right)$, considered as the critical factor for most electrophilic reactions $[8,11]$, was poorly correlated with sensitization potential. These results suggest the skin sensitization mechanism of those compounds may result from several steps but not a directly electrophilic reaction.

The $\epsilon_{\mathrm{HOMO}}$ dependent results implied that a process of losing electron may be involved in the activation of those sensitizers. Those compounds may be activated via an autoxidation mechanism to further interact with skin proteins as prehaptens. In addition, the mechanisms where these chemicals directly react with free radical of skin proteins also should be considered [42]. In the present study, we proposed that two potential pathways could lead these compounds to cause skin sensitization as shown in Scheme 1 [42]. In the first pathway (Scheme 1a), an aniline (or a phenol) is readily oxidized to a radical cation through loss of an electron at the aromatic ring [43] and forms two possible reactive intermediates. A protein-associated sulfhydryl radical then attacks the aromatic of the radical cation to form a covalent bond at the orth- or para-position. Or, the reactive intermediates bind to nucleophilic moieties on proteins through the Michael addition. The second pathway corresponds to what Lepoittevin defined as a direct haptenation route [44], whereby attack of a protein associated sulfhydryl radical on the ring gives an intermediate radical (Scheme 1b).

A compound with lower energy of HOMO appears either more stable or less reactive when reacting with a protein associated sulfhydryl radical [24]. Therefore, as shown in Figure 3, the sensitizers e.g., the aniline (CAS: 62-53-3) and the eugenol (CAS: 97-53-0) equipped with higher $\epsilon_{\mathrm{HOMO}}$ values can lose electron(s) more easily to form radical cation intermediates than nonsensitizers (e.g., 2-fluoro-5-nitroaniline (CAS: 369-368), 4-aminobenzoic acid (CAS: 150-13-0), salicylic acid (CAS: 69-72-7), and 3-hydroxy-4-nitrobenzoic acid (CAS: 619-14-7)). We noted that the non-sensitizers of anilines and phenols are those that have the electron withdrawing groups attached to the aromatic ring, such as $-\mathrm{F},-\mathrm{NO}_{2}$, $-\mathrm{COOH}$. This implies that introduction of electron withdrawing groups to the aromatic ring of anilines or phenols may be one of the effective ways to reduce the potency of skin sensitizers. 
(a)

The pro-oxidation mechanism

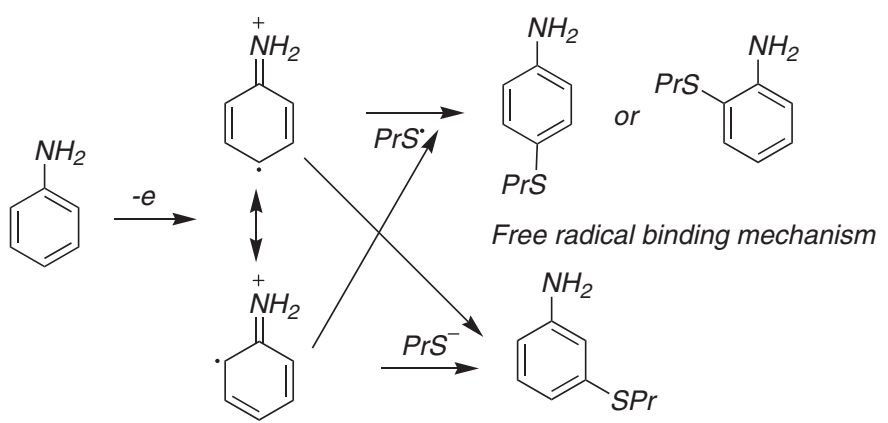

(b) The direct reaction mechanism

Pro-Michael addition mechanism<smiles>Nc1ccccc1-c1ccc(F)cc1</smiles>

Scheme 1 Reaction mechanisms of anilines binding to protein. (a) The pro-oxidation mechanism. (b) The direct reaction mechanism.

Predicting skin sensitization potentials of a subset of FDA approved drugs with aniline and phenol groups

There are no effective tools to predict the skin sensitization potential of drugs, because drug-induced skin reactions may be caused by several mechanisms either single or mixed [27]. The skin sensitization, in the context, refers to T-cell mediated sensitization (type IV allergy). The reaction of chemicals with proteins was recognized as one of the necessary process of the T-cell mediated sensitization [23]. The in silico mechanistic models may offer valuable insights into better understanding the initiation of drug induced allergies.

We collected 53 drugs containing aniline and/or phenol moieties from the DrugBank database. The information of these 53 compounds is also available in the Additional file 1. These FDA approved drugs were then analyzed to filter out those with structural alerts of skin sensitization. The sulfonamide drugs were also removed due to they have different mechanisms of action. For example, sulfamethoxazole (DrugBank ID: DB01015) can

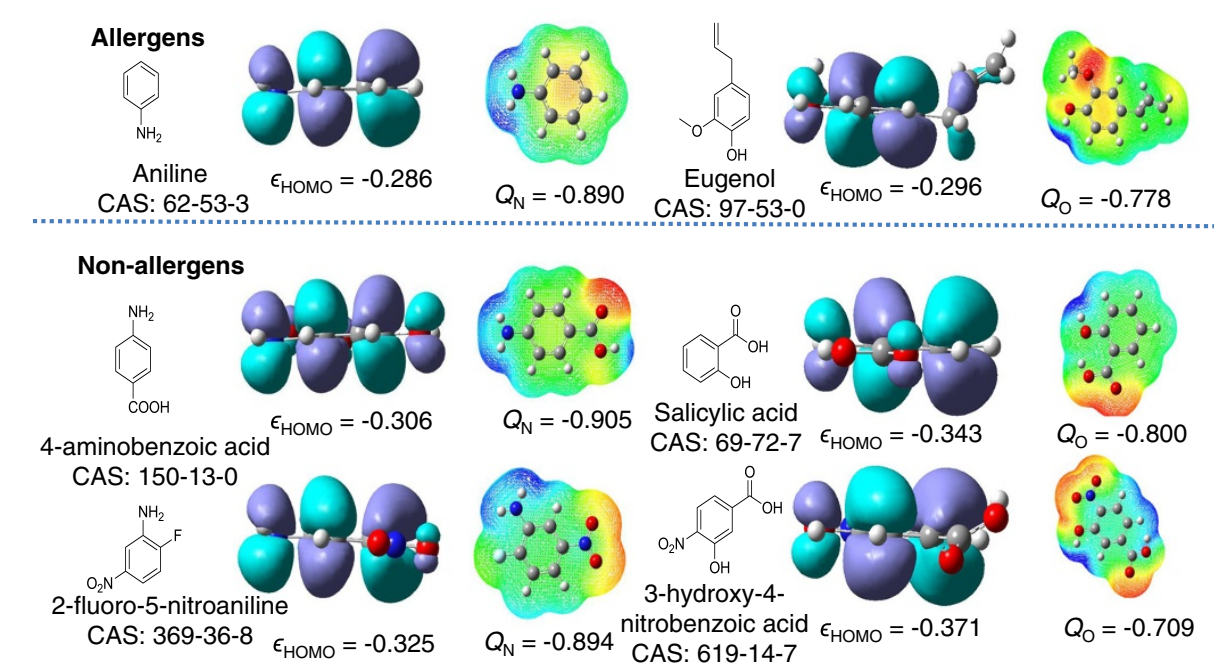

Figure 3 The structures, energies and shapes of HOMO, and charge density of representative anilines and phenols. Anilines (left column: aniline, 4-aminobenzoic acid, and 2-fluoro-5-nitroaniline); Phenols (right column: eugenol, salicylic acid, and 3-hydroxy-4-nitrobenzoic acid). The unit of the energy is hartree. 
Table 2 Prediction of skin sensitization potential for 6 FDA approved drugs that have side effect of allergic dermatitis reported in MetaADEDB database

\begin{tabular}{|c|c|c|c|c|c|}
\hline & DrugBank ID & Name & $P$ & Prediction $^{a}$ & MetaADEDB $^{b}$ \\
\hline 1 & DB00279 & Liothyronine & -0.001 & N & Y \\
\hline 2 & DB01407 & Clenbuterol & 0.589 & Y & Y \\
\hline 3 & DB00250 & Dapsone & 0.700 & Y & Y \\
\hline 4 & DB00295 & Morphine & 0.580 & Y & Y \\
\hline 5 & DB00327 & Hydromorphone & 0.595 & Y & Y \\
\hline 6 & DB00481 & Raloxifene & 0.977 & Y & Y \\
\hline
\end{tabular}

${ }^{a} \mathrm{~A}$ drug is predicted as an sensitizer if its $P$ value is greater than 0.50 ; Otherwise, as a non-sensitizer. ${ }^{b}$ Compounds having the keywords "allergic dermatitis" in their side effect reports in the MetaADEDB database are indicated as sensitizers. $Y$ : Sensitizer; N: Non-sensitizer.

be oxidized to a hydroxylamine metabolite and subsequently form a reactive nitroso intermediate by autooxidation that enables it to react with skin proteins [45]. Finally, twenty six compounds were obtained and their skin sensitization potential was predicted by our model. Among these 26 compounds, six of them were reported to be able to induce "allergic dermatitis" according to the side effect information in MetaADEDB database (Table 2). Interestingly, our results showed that five of them, e.g. Clenbuterol, Dapsone, Morphine, Hydromorphone and Raloxifene were correctly predicted as sensitizers (Table 2) as their $P$ values are greater than the threshold 0.50. In addition, allergic dermatitis is a rare side effect of Liothyronine according to the information from https://www.universaldrugstore.com/medications/ Liothyronine/side-effects. However, users should be cautious that there is no label for drugs not causing "allergic dermatitis", thus it is hard to find a negative set in FDA approved drugs to further evaluate our model.

\section{Conclusion}

This study has demonstrated how quantum chemical calculations can be utilized to predict skin sensitization potential and to infer the reaction mechanism for a class of mechanistically hard-to-be-classified chemicals containing aniline and phenol moieties. The outcomes emphasized that the energy of highest occupied molecular orbital plays an important role for predicting skin sensitization potential of these compounds, indicating the activation process occurred via either autoxidation or direct reaction with free radical. Our model was further applied to predict the allergenic potential of the approved drugs containing aniline and/or phenol moieties. Several of these drugs were identified as sensitizers and the prediction agreed well with their "allergic dermatitis" side effect. Thus, the data indicate that our newly developed in silico algorithm shows promise as a preclinical risk assessment tool for screening allergenic potential.

Again, we should point out that skin allergic reactions are not commonly seen for drugs given via the oral route. Though they may share similar mechanisms, caution should be taken when extrapolating our model from skin sensitization potential for topically applied chemicals to predict "allergic potential" of drugs.

\section{Additional file}

Additional file 1: Predicted values and experimental data of reported chemicals and FDA approved drugs that contain aniline and/or phenol moieties.

\section{Abbreviations}

ACD: Allergic contact dermatitis; AM1: Austin model 1; CAS: Chemical abstracts service; EC3: Effective concentration for a three-fold proliferation of lymph node cells; FDA: Food and drug administration; GPMT: Guinea pig maximization test; HOMO: Highest occupied molecular orbital; LLNA: The murine-based local lymph node assay; LUMO: Lowest unoccupied molecular orbital; MA: Michael acceptors; SAR: Structure-activity relationship; SMILES: Simplified molecular-input line-entry system; $\mathrm{SN}_{2}$ : A kind of nucleophilic substitution reaction mechanism; $S_{N} A r$ : Nucleophilic aromatic substitution.

\section{Competing interests}

The authors declare that they have no competing interests.

\section{Authors' contributions}

$\mathrm{OQ}$ and LW carried out the experiments; OQ, LW and X-QX designed the study and carried out the analysis. OQ, LW, YM and X-QX interpreted the data and drafted the manuscript. X-QX supervised the progress and critically revised the manuscript. All authors read and approved the final manuscript.

\section{Acknowledgements}

OQ would like to acknowledge the support from the National Natural Science Foundation of China (NSFC21202201).

\section{Disclaimer}

The mention of commercial products, their sources, or their use in connection with material reported herein is not to be construed as either an actual or implied endorsement of such products by the Department of Health and Human Services. The findings and conclusions in this article have not been formally disseminated by the Food and Drug Administration and should not be considered to represent any agency determination or policy.

\section{Release of copyright permission}

There is no copyright in U.S. Government work (per 17 U.S.C. 105), and the work I am providing is a U.S. Government work. 


\section{Author details}

${ }^{1}$ Department of Pharmaceutical Sciences, Computational Chemical Genomics Screening Center, School of Pharmacy, NIH National Center, of Excellence for Computational Drug Abuse Research, Drug Discovery Institute, Pittsburgh, PA 15261, USA. ²Department of Computational and Systems Biology, University of Pittsburgh, Pittsburgh, PA 15261, USA. ${ }^{3}$ Division of Biology, Office of Science and Engineering Laboratories, Center for Devices and Radiobiological Health, US Food and Drug Administration, Silver Spring, MD 20993, USA. ${ }^{4}$ College of Pharmacy, Third Military Medical University, Chongqing 400038, China.

Received: 31 March 2014 Accepted: 20 November 2014

Published: 24 December 2014

\section{References}

1. Lushniak BD: Occupational contact dermatitis. Dermatol Ther 2004, 17(3):272-277.

2. Wizemann T: Public Health Effectiveness of the FDA 510(k) Clearance Process: Measuring Postmarket Performance and Other Select Topics: Workshop Report (2011); 2011.

3. Basketter DA, Scholes EW: Comparison of the local lymph-node assay with the guinea-pig maximization test for the detection of a range of contact allergens. Food Chem Toxicol 1992, 30(1):65-69.

4. Uter W, Johansen JD, Borje A, Karlberg AT, Liden C, Rastogi S, Roberts D, White IR: Categorization of fragrance contact allergens for prioritization of preventive measures: clinical and experimental data and consideration of structure-activity relationships. Contact Dermatitis 2013, 69(4):196-230.

5. Teubner W, Mehling A, Schuster PX, Guth K, Worth A, Burton J, van Ravenzwaay $B$, Landsiedel $R$ : Computer models versus reality: how well do in silico models currently predict the sensitization potential of a substance. Regul Toxicol Pharmacol 2013, 67(3):468-485.

6. Enoch SJ, Cronin MT, Schultz TW, Madden JC: An evaluation of global QSAR models for the prediction of the toxicity of phenols to Tetrahymena pyriformis. Chemosphere 2008, 71(7):1225-1232

7. Landsteiner $\mathrm{K}$, Jacobs J: Studies on the sensitization of animals with simple chemicals compounds. II. J Exp Med 1936, 64:625-639.

8. Aptula AO, Roberts DW: Mechanistic applicability domains for nonanimalbased prediction of toxicological end points: general principles and application to reactive toxicity. Chem Res Toxicol 2006, 19(8):1097-1105.

9. Divkovic M, Pease CK, Gerberick GF, Basketter DA: Hapten-protein binding: from theory to practical application in the in vitro prediction of skin sensitization. Contact Dermatitis 2005, 53(4):189-200.

10. Aleksic M, Thain E, Roger D, Saib O, Davies M, Li J, Aptula A, Zazzeroni R: Reactivity profiling: covalent modification of single nucleophile peptides for skin sensitization risk assessment. Toxicol Sci 2009, 108(2):401-411.

11. Enoch SJ, Madden JC, Cronin MTD: Identification of mechanisms of toxic action for skin sensitisation using a SMARTS pattern based approach. Sar Qsar Environ Res 2008, 19(5-6):555-578.

12. Delaine T, Hagvall L, Rudbäck J, Luthman K, Karlberg A-T: Skin sensitization of epoxyaldehydes: importance of conjugation. Chem Res Toxicol 2013, 26(674):684

13. Enoch SJ, Roberts DW: Predicting skin sensitization potency for michael acceptors in the LLNA using quantum mechanics calculations. Chem Res Toxicol 2013, 26(5):767-774

14. Roberts DW, Aptula AO, Patlewicz GY: Chemistry-based risk assessment for skin sensitization: quantitative mechanistic modeling for the SNAr domain. Chem Res Toxicol 2011, 24(7):1003-1011.

15. Natsch A, Gfeller H, Haupt T, Brunner G: Chemical reactivity and skin sensitization potential for Benzaldehydes: can schiff base formation explain everything? Chem Res Toxicol 2012, 25(10):2203-2215.

16. Bergström MA, Luthman K, Nilsson JLG, Karlberg A-T: Conjugated dienes as prohaptens in contact allergy: in Vivo and in Vitro studies of structure activity relationships, sensitizing capacity, and metabolic activation. Chem Res Toxicol 2006, 19(6):760-769.

17. Bergström MA, Andersson SI, Broo K, Luthman K, Karlberg A-T: Oximes: metabolic activation and structure - allergenic activity relationships. J Med Chem 2008, 51(8):2541-2550.

18. Patlewicz GY, Basketter DA, Smith Pease CK, Wilson K, Wright ZM, Roberts DW, Bernard G, Arnau EG, Lepoittevin J-P: Further evaluation of quantitative structure-activity relationship models for the prediction of the skin sensitization potency of selected fragrance allergens. Contact Dermatitis 2004, 50(2):91-97.

19. Niklasson IB, Delaine T, Luthman K, Karlberg A-T: Impact of a heteroatom in a structure - activity relationship study on analogues of Phenyl Glycidyl Ether (PGE) from epoxy resin systems. Chem Res Toxicol 2011, 24(4):542-548

20. Itoh M: Sensitization potency of some phenolic compounds-with special emphasis on the relationship between chemical structure and allergenicity. J Dermatol 1982, 9(3):223-233.

21. Malkowski J, Klenieswka D, Maibach H: Relationship between chemical structure and allergenicity: aromatic amines. Derm Beruf Umwelt 1983, 31(2):48-50.

22. Kleniewska $\mathrm{D}$, Maibach $\mathrm{H}$ : Allergenicity of aminobenzene compounds: structure-function relationships. Derm Beruf Umwelt 1980, 28(1):11-13.

23. Payne MP, Walsh PT: Structure-activity-relationships for skin sensitization potential - development of structural alerts for use in knowledge-based toxicity prediction systems. J Chem Inf Comput Sci 1994, 34(1):154-161.

24. Aptula AO, Enoch SJ, Roberts DW: Chemical mechanisms for skin sensitization by aromatic compounds with hydroxy and amino groups. Chem Res Toxicol 2009, 22(9):1541-1547.

25. Patlewicz G, Roberts DW, Uriarte E: A comparison of reactivity schemes for the prediction skin sensitization potential. Chem Res Toxicol 2008, 21(2):521-541.

26. Cuyper C, Goeteyn M: Systemic contact dermatitis from subcutaneous hydromorphone. Contact Dermatitis 1992, 27(4):220-223.

27. Lee A, Thomson J: Drug-induced skin reactions. Pharmaceut J 1999 2006, 262:357-362.

28. Gerberick GF, Vassallo JD, Bailey RE, Chaney JG, Morrall SW, Lepoittevin JP. Development of a peptide reactivity assay for screening contact allergens. Toxicol Sci 2004, 81(2):332-343.

29. Estrada E, Patlewicz G, Gutierrez Y: From knowledge generation to knowledge archive. a general strategy using TOPS-MODE with DEREK to formulate new alerts for skin sensitization. J Chem Inf Comput Sci 2004, 44(2):688-698

30. Barratt MD, Langowski JJ: Validation and subsequent development of the DEREK skin sensitization rulebase by analysis of the BgVV list of contact allergens. J Chem Inf Comput Sci 1999, 39(2):294-298.

31. Schneider K, Akkan Z: Quantitative relationship between the local lymph node assay and human skin sensitization assays. Regul Toxicol Pharmacol 2004, 39(3):245-255.

32. Roberts DW, Patlewicz G, Kern PS, Gerberick F, Kimber I, Dearman RJ, Ryan CA, Basketter DA, Aptula AO: Mechanistic applicability domain classification of a local lymph node assay dataset for skin sensitization. Chem Res Toxicol 2007, 20(7):1019-1030.

33. Miller MD, Yourtee DM, Glaros AG, Chappelow CC, Eick JD, Holder AJ: Quantum mechanical structure-activity relationship analyses for skin sensitization. J Chem Inf Model 2005, 45(4):924-929.

34. Kern PS, Gerberick GF, Ryan CA, Kimber I, Aptula A, Basketter DA: Local lymph node data for the evaluation of skin sensitization alternatives: a second compilation. Dermatitis 2010, 21(1):8-32.

35. Estrada E, Patlewicz G, Chamberlain M, Basketter D, Larbey S: Computeraided knowledge generation for understanding skin sensitization mechanisms: the TOPS-MODE approach. Chem Res Toxicol 2003, 16(10):1226-1235.

36. Basketter DA: Skin sensitization: strategies for the assessment and management of risk. Br J Dermatol 2008, 159(2):267-273.

37. Frisch MJ, Trucks GW, Schlegel HB, Scuseria GE, Robb MA, Cheeseman JR, Montgomery JA Jr, Vreven T, Kudin KN, Burant JC, Millam JM, lyengar SS, Tomasi J, Barone V, Mennucci B, Cossi M, Scalmani G, Rega N, Petersson GA, Nakatsuji H, Hada M, Ehara M, Toyota K, Fukuda R, Hasegawa JIM, Nakajima T, Honda Y, Kitao O, Nakai H: Gaussian 03. In., Revision C.02. Wallingford, CT: Gaussian, Inc; 2003

38. Dewar MJS, Zoebisch EG, Healy EF, Stewart JJP: Development and use of quantum mechanical molecular models. 76. AM1: a new general purpose quantum mechanical molecular model. J Am Chem Soc 1985, 107(13):3902-3909

39. Enoch SJ, Roberts DW, Cronin MTD: Mechanistic category formation for the prediction of respiratory sensitization. Chem Res Toxicol 2010, 23(10):1547-1555.

40. R Development Core Team: R: A Language and Environment for Statistical Computing. Vienna, Austria: R Foundation for Statistical Computing; 2010. Retrieved from http://www.R-project.org. 
41. Enoch SJ, Cronin MT, Schultz TW, Madden JC: Quantitative and mechanistic read across for predicting the skin sensitization potential of alkenes acting via Michael addition. Chem Res Toxicol 2008, 21(2):513-520.

42. Karlberg AT, Bergstrom MA, Borje A, Luthman K, Nilsson JLG: Allergic contact dermatitis-formation, structural requirements, and reactivity of skin sensitizers. Chem Res Toxicol 2008, 21(1):53-69.

43. Storle $C$, Eyer P: Reactions of the wurster blue radical cation with thiols, and some properties of the reaction-products. Chem Biol Interact 1991, 78(3):333-346

44. Lepoittevin JP: Metabolism versus chemical transformation or pro- versus prehaptens? Contact Dermatitis 2006, 54(2):73-74.

45. Choquet-Kastylevsky G, Vial T, Descotes J: Allergic adverse reactions to sulfonamides. Curr Allergy Asthma Rep 2002, 2(1):16-25.

doi:10.1186/2050-6511-15-76

Cite this article as: Ouyang et al:: Modeling skin sensitization potential of mechanistically hard-to-be-classified aniline and phenol compounds with quantum mechanistic properties. BMC Pharmacology and Toxicology 2014 15:76

\section{Submit your next manuscript to BioMed Central and take full advantage of:}

- Convenient online submission

- Thorough peer review

- No space constraints or color figure charges

- Immediate publication on acceptance

- Inclusion in PubMed, CAS, Scopus and Google Scholar

- Research which is freely available for redistribution 